\title{
Road Geometry Estimation and Vehicle Tracking using a Single Track Model
}

\author{
Christian Lundquist and Thomas Schön
}

\section{Linköping University Post Print}

N.B.: When citing this work, cite the original article.

(C2010 IEEE. Personal use of this material is permitted. However, permission to reprint/republish this material for advertising or promotional purposes or for creating new collective works for resale or redistribution to servers or lists, or to reuse any copyrighted component of this work in other works must be obtained from the IEEE.

Christian Lundquist and Thomas Schön, Road Geometry Estimation and Vehicle Tracking using a Single Track Model, 2008, 2008 IEEE Intelligent Vehicles Symposium, 144-149. http://dx.doi.org/10.1109/IVS.2008.4621231

Postprint available at: Linköping University Electronic Press http://urn.kb.se/resolve?urn=urn:nbn:se:liu:diva-44271 


\title{
Road geometry estimation and vehicle tracking using a single track model
}

\author{
Christian Lundquist and Thomas B. Schön \\ Division of Automatic Control \\ Linköping University \\ SE-581 83 Linköping, Sweden \\ Email: \{lundquist, schon\}@isy.liu.se
}

\begin{abstract}
This paper is concerned with the, by now rather well studied, problem of integrated road geometry estimation and vehicle tracking. The main differences to the existing approaches are that we make use of an improved host vehicle model and a new dynamic model for the road. The problem is posed within a standard sensor fusion framework, allowing us to make good use of the available sensor information. The performance of the solution is evaluated using measurements from real and relevant traffic environments from public roads in Sweden. The experiments indicates that the gain in using the extended host vehicle model is most prominent when driving on country roads without any vehicles in front.
\end{abstract}

\section{INTRODUCTION}

We are concerned with the, by now rather well studied, problem of automotive sensor fusion. More specifically, we consider the problem of integrated road geometry estimation and vehicle tracking making use of an improved host vehicle model. The overall aim in the present paper is to extend the existing results to a more complete treatment of the problem by making better use of the available information.

In order to facilitate a systematic treatment of this problem we need dynamical models for the host vehicle, the road and the leading vehicles. These models are by now rather well understood. However, in studying sensor fusion problems this information tends not to be used as much as it could. Dynamic vehicle modelling is a research field in itself and a solid treatment can be found in for example [13], [16]. The leading vehicles can be successfully modelled using the geometrical constraints and their derivatives w.r.t. time. Finally, dynamic models describing the road are rather well treated, see e.g., [4]-[6]. The resulting state-space model, including host vehicle, road and leading vehicles, can then be written in the form

$$
\begin{aligned}
x_{t+1} & =f\left(x_{t}, u_{t}\right)+w_{t}, \\
y_{t} & =h\left(x_{t}, u_{t}\right)+e_{t},
\end{aligned}
$$

where $x_{t}$ denotes the state vector, $u_{t}$ denotes the input signal, $w_{t}$ denotes the process noise, $y_{t}$ denotes the measurements and $e_{t}$ denotes the measurement noise. Once we have derived a model in the form (1) the problem has been transformed into a standard nonlinear estimation problem. This problem has been extensively studied within the control and the target tracking communities for many different application areas. There are many different ways to solve it, including the popular Extended Kalman Filter (EKF), the particle filter and the Unscented Kalman Filter (UKF), see e.g., [1], [12] for more information on this topic.

As mentioned above, the problem studied in this paper is by no means new, see e.g., [4], [5] for some early work without using the motion of the leading vehicles. These papers are still very interesting reading and contain much of the underlying ideas that are being used today. It is also interesting to note that the importance of sensor fusion was stressed already in these early papers. The next step in the development was to introduce a radar sensor as well. The idea was that the motion of the leading vehicles reveals information about the road geometry [9], [10], [21]. Hence, if the leading vehicles can be accurately tracked, their motion can be used to improve the road geometry estimates, computed using only information about the host vehicle motion and information about the road inferred from a vision sensor. This idea has been further refined and developed in [6], [8], [19]. However, the dynamic model describing the host vehicle used in all of these later works were significantly simplified as compared to the one used in [3]-[5]. It consists of 2 states, the distance from the host vehicle to the white lane and the heading (yaw) angle of the host vehicle. Hence, it does not contain any information about the host vehicles velocity vector. Information of this kind is included in the host vehicle model employed in the present paper.

The main contribution of this work is to pose and solve a sensor fusion problem that makes use of the information from all the available sensors. This is achieved by unifying the ideas in the above referenced papers. The host vehicle is modelled in more detail, it bears most similarity to the model used in [4], [5]. Furthermore, we include the motion of the leading vehicles, using the idea introduced in [21]. The resulting sensor fusion problem provides a rather systematic treatment of the information from the sensors measuring the host vehicle motion (inertial sensors, steering wheel sensors and wheel speed sensors) and the sensors measuring the vehicle surroundings (vision and radar).

It is also shown how the suggested sensor fusion approach performs in practise, by evaluating it using measurements from real and relevant traffic environments from public roads in Sweden.

\section{Dynamic Models}

In this section we will derive the differential equations describing the motion of the host vehicle (Section II-B), the road (Section II-C) and the leading vehicles (Section II-D), also referred to as targets. However, before we embark on deriving these equations we introduce the overall geometry and some notation in Section II-A.

\section{A. Geometry and Notation}

The coordinate frames describing the host vehicle and one leading vehicle are defined in Fig. 1. The inertial reference frame is denoted by $R$ and its origin is $O$, the other frames are denoted by $L_{i}$, with origin in $P_{i} . P_{1}$ and $P_{2}$ are attached to the rear and front wheel axle of the host vehicle, respectively. $P_{3}$ is used to describe the road and $P_{4}$ is located in the center of gravity $(\mathrm{CoG})$ for the host vehicle. Furthermore, $L_{S n}$ is associated to the observed leading vehicle $n$, with 


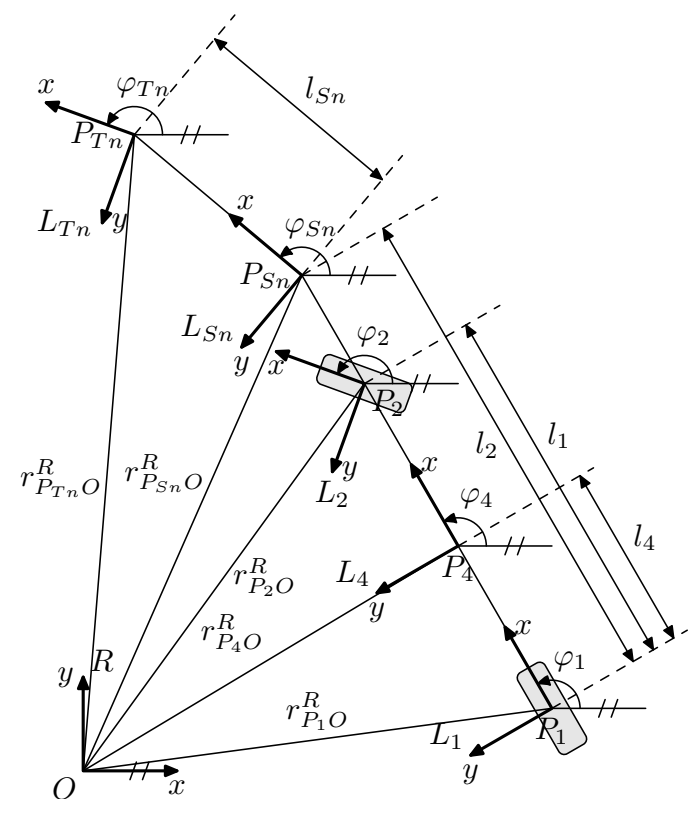

Fig. 1. Coordinate frames describing the host vehicle and one leading vehicle $T n$.

$P_{S n}$ at the sensor of the host vehicle. Finally, $L_{T n}$ is also associated with the observed leading vehicle $n$, but its origin $P_{T n}$ is located at the leading vehicle.

\section{B. Host Vehicle}

We will only be concerned with the host vehicle motion during normal driving situations and not at the wheel-track adhesion limit. This implies that the single track model [16] is sufficient for the present purposes. The geometry of the single track model with slip angles is shown in Fig. 2. It is here worth to point out that the velocity vector of the host vehicle is typically not in the same direction as the longitudinal axis of the host vehicle. Instead the vehicle will move along a path at an angle $\beta$ with the longitudinal direction of the vehicle. This angle $\beta$ is referred to as the float angle [17] or vehicle body side slip angle [13].

The slip angle $\alpha_{i}$ is defined as the angle between the central axis of the wheel and the path along which the wheel moves. The phenomenon of side slip is mainly due to the lateral elasticity of the tire. For reasonably small slip angles, at maximum $3 \mathrm{deg}$, it is a good approximation to assume that the lateral friction force of the tire $F_{i}$ is proportional to the slip angle,

$$
F_{i}=C_{\alpha i} \alpha_{i}
$$

The parameter $C_{\alpha i}$ is called cornering stiffness and describes the cornering behaviour of the tire. A deeper analysis of slip angles can be found in e.g., [16]. Furthermore, the front wheel angle $\delta_{F}$, i.e. the angle between the longitudinal direction of the front wheel and the longitudinal axis of the host vehicle, is defined as

$$
\delta_{F} \triangleq \varphi_{2}-\varphi_{1} \text {. }
$$

Following this introduction to the host vehicle geometry we are now ready to give an expression of the host vehicle's velocity vector, resolved in the inertial frame $R$,

$$
\begin{gathered}
\dot{x}_{P_{4} O}^{R}=v_{x} \cos \left(\varphi_{1}+\beta\right), \\
\dot{y}_{P_{4} O}^{R}=v_{x} \sin \left(\varphi_{1}+\beta\right),
\end{gathered}
$$

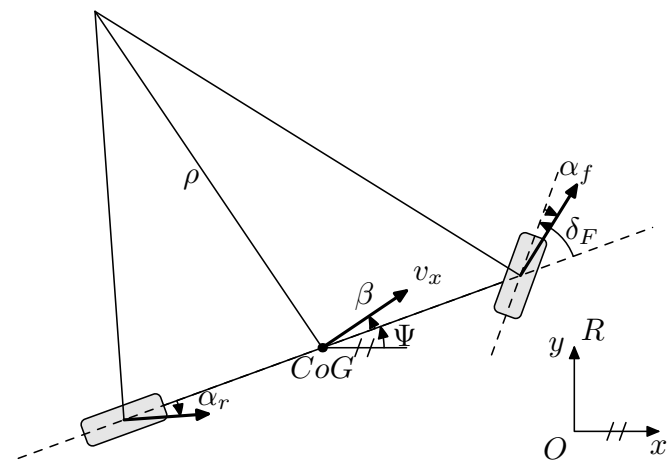

Fig. 2. Illustration of the geometry for the single track model, describing the motion of the host vehicle. The host vehicle velocity vector $v_{x}$ is defined from the CoG and its angle to the longitudinal axis of the vehicle is denoted by $\beta$, referred to as the float angle or vehicle body side slip angle. Furthermore, the slip angles are referred to as $\alpha_{f}$ and $\alpha_{r}$. The front wheel angle is denoted by $\delta_{F}$ and the current radius is denoted by $\rho$.

which is governed by the yaw angle $\varphi_{1}$ and the float angle $\beta$. Hence, in order to find the state-space model we are looking for, we need the differential equations describing the evolution of these angles over time. These equations are well-known from the literature, see e.g., [13], hence we give them here without derivation

$$
\begin{aligned}
\ddot{\varphi}_{1} & =-\dot{\varphi}_{1} \frac{C_{\alpha f}\left(l_{1}-l_{4}\right)^{2} \cos \delta_{F}+C_{\alpha r} l_{4}^{2}}{J v_{x}}+\frac{\left(l_{1}-l_{4}\right) C_{\alpha f} \tan \delta_{F}}{J} \\
& +\beta \frac{\left(-\left(l_{1}-l_{4}\right) C_{\alpha f} \cos \delta_{F}+l_{4} C_{\alpha r}\right)}{J} \\
\dot{\beta} & =\beta \frac{-C_{\alpha f} \cos \delta_{F}-C_{\alpha r}-\dot{v}_{x} m}{m v_{x}}+\frac{C_{\alpha f} \sin \delta_{F}}{m v_{x}} \\
& -\dot{\varphi}_{1}\left(1+\frac{C_{\alpha f}\left(l_{1}-l_{4}\right) \cos \delta_{F}-C_{\alpha r} l_{4}}{v_{x}^{2} m}\right)
\end{aligned}
$$

where $m$ denotes the mass of the vehicle and $J$ denotes the moment of inertia of the vehicle about its vertical axis in the center of gravity.

\section{Road}

The essential component in describing the road geometry is the curvature $c$, which is defined as the curvature of the white line to the left of the host vehicle. An overall description of the road geometry is given in Fig. 3. In order to model the road curvature we introduce the road coordinate frame $L_{3}$, with its origin $P_{3}$ on the white line to the left of the host vehicle, with $x^{L_{1}}=l_{2}$. This implies that the frame $L_{3}$ is moving with the $x$-axis of the host vehicle. The angle of the $L_{3}$ frame $\varphi_{3}$ is defined as the tangent of the road in $x^{L_{3}}=0$, see Fig. 4. This implies that $\varphi_{3}$ is defined as

$$
\varphi_{3} \triangleq \varphi_{1}+\delta_{r}
$$

where $\delta_{r}$ is the angle between the tangent of the road curvature and the longitudinal axis of the host vehicle, i.e.,

$$
\delta_{r}=\beta+\delta_{R} .
$$

Here, $\delta_{R}$ is the angle between the host vehicles direction of motion (velocity vector) and the road curvature tangent. Hence, inserting (8) into (7) we have

$$
\varphi_{3}=\varphi_{1}+\beta+\delta_{R} .
$$

Furthermore, the road curvature $c$ is typically parameterized according to

$$
c\left(x_{c}\right)=c_{0}+c_{1} x_{c}
$$




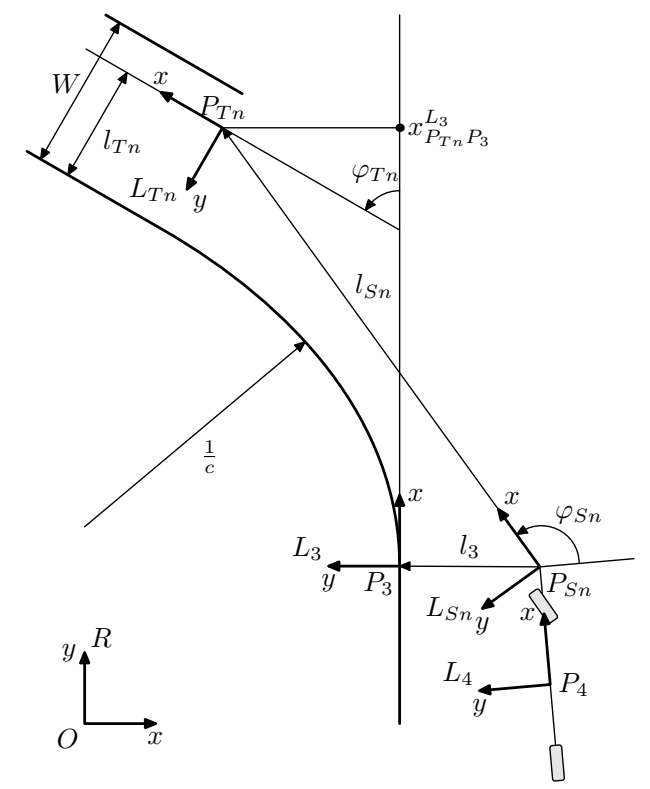

Fig. 3. Relations between the leading vehicles $T n$, the host vehicle and the road. The distance between the host vehicle path and the white lane to its left (where the road curvature is defined) is $l_{3}$. The lane width is $W$.

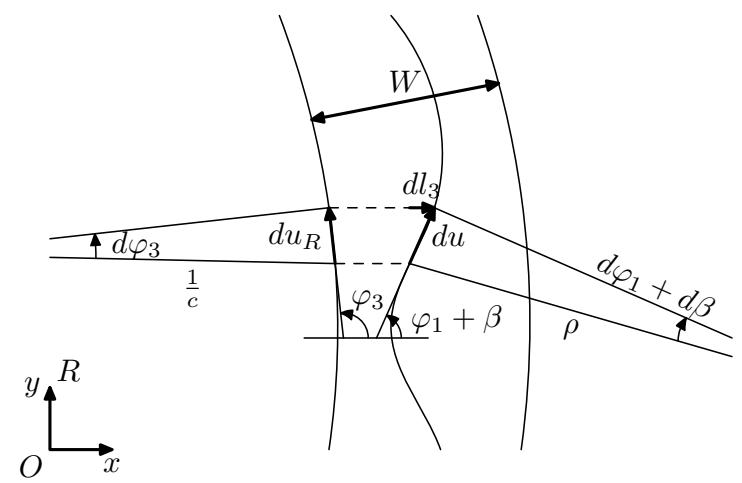

Fig. 4. Representation of the road curvature $c_{0}$, the radius of the (driven) path $\rho$ and the angles $\delta_{R}=\varphi_{3}-\left(\varphi_{1}+\beta\right)$. The lane width is $W$.

where $x_{c}$ is the position along the road in a road aligned coordinate frame. Furthermore, $c_{0}$ describes the local curvature at the host vehicle position and $c_{1}$ is the distance derivative (hence, the rate of change) of $c_{0}$. It is common to make use of a road aligned coordinate frame in deriving an estimator for the road geometry, a good overview of this approach is given in [6]. However, we will make use of a Cartesian coordinate frame. A good polynomial approximation of the shape of the road curvature is given by

$$
y^{L_{3}}=\frac{c_{0}}{2}\left(x^{L_{3}}\right)^{2}+\frac{c_{1}}{6}\left(x^{L_{3}}\right)^{3},
$$

see e.g., [4], [6]. The following dynamic model is often used for the road

$$
\dot{c}_{0}=v_{x} c_{1}, \quad \dot{c}_{1}=0,
$$

which in discrete time can be interpreted as a velocity dependent integration of white noise. It is interesting to note that (12) reflects the way in which roads are commonly built [4]. However, we will now derive a new dynamic model for the road that makes use of the road geometry introduced above.
1) Road Angle: Assume that $d u_{R}$ is a part of the road curvature or an arc of the road circle with the angle $d \varphi_{3}$, see Fig. 4. A segment of the road circle can be described as

$$
d u_{R}=\frac{1}{c_{0}} \cdot d \varphi_{3},
$$

which after division with the differential w.r.t. time $d t$ is given by

$$
\frac{d u_{R}}{d t}=\frac{1}{c_{0}} \cdot \frac{d \varphi_{3}}{d t}, \quad v_{x}=\frac{1}{c_{0}} \cdot \dot{\varphi}_{3},
$$

where we have assumed that $\frac{d u_{R}}{d t}=v_{x} \cos \delta_{R} \approx v_{x}$. Reordering the equation and using the derivative of (9) to substitute $\varphi_{3}$ yields

$$
\dot{\delta}_{R}=c_{0} v_{x}-\left(\dot{\varphi}_{1}+\dot{\beta}\right) .
$$

A similar relation has been used in [4], [14].

2) Road Curvature: Differentiating (15) w.r.t. time gives

$$
\ddot{\delta}_{R}=\dot{c}_{0} v_{x}+c_{0} \dot{v}_{x}-\ddot{\varphi}_{1}-\ddot{\beta},
$$

from which we have

$$
\dot{c}_{0}=\frac{\ddot{\delta}_{R}+\ddot{\varphi}_{1}+\ddot{\beta}-c_{0} \dot{v}_{x}}{v_{x}} .
$$

Assume $\ddot{\delta}_{R}=0$, inserting $\ddot{\varphi}_{1}$ which was given in (5), and differentiating $\dot{\beta}$, from (6), w.r.t. time yields

$$
\begin{gathered}
\dot{c}_{0}=\frac{1}{\left(J m^{2} v_{x}\right)^{4}}\left(C_{\alpha r}^{2}\left(J+l_{4}^{2} m\right)\left(-\dot{\varphi}_{1} l_{4}+\beta v_{x}\right)\right. \\
+C_{\alpha f}^{2}\left(J+\left(l_{1}-l_{4}\right)^{2} m\right)\left(\dot{\varphi}_{1}\left(l_{1}-l_{4}\right)+\left(\beta-\delta_{F}\right) v_{x}\right) \\
\quad+C_{\alpha r} J m\left(-3 \dot{\varphi}_{1} \dot{v}_{x} l_{4}+3 \beta \dot{v}_{x} v_{x}+\dot{\varphi}_{1} v_{x}^{2}\right) \\
\quad+\dot{v}_{x} J m^{2} v_{x}\left(2 \beta \dot{v}_{x}+v_{x}\left(\dot{\varphi}_{1}-c_{0} v_{x}\right)\right) \\
+C_{\alpha f}\left(C_{\alpha r}\left(J+l_{4}\left(-l_{1}+l_{4}\right) m\right)\left(\dot{\varphi}_{1} l_{1}-2 \dot{\varphi}_{1} l_{4}+2 \beta v_{x}-\delta_{F} v_{x}\right)\right. \\
\left.\left.+J m\left(3 \dot{\varphi}_{1} \dot{v}_{x}\left(l_{1}-l_{4}\right)+\left(3 \beta-2 \delta_{F}\right) \dot{v}_{x} v_{x}+\left(\dot{\delta}_{F}+\dot{\varphi}_{1}\right) v_{x}^{2}\right)\right)\right)
\end{gathered}
$$

3) Distance Between the Host Vehicle Path and the White Line: Assume a small arc $d u$ of the circumference describing the host vehicle's curvature, see Fig. 4. The angle between the host vehicle and the road is $\delta_{R}$, thus

$$
d l_{3}=d u \sin \delta_{R}, \quad i_{3}=v_{x} \sin \delta_{R} .
$$

\section{Leading Vehicles}

1) Geometric Constraints: The leading vehicles are also referred to as targets $T n$. The coordinate frame $L_{T n}$ moving with target $n$ is located in $P_{T n}$, as we saw in Fig. 3. It is assumed that the leading vehicles are driving on the road. More specifically, it is assumed that they are following the road curvature and thus that their heading is the same as the tangent of the road.

For each target $T n$, there exists a coordinate frame $L_{S n}$, with its origin $P_{S n}$ at the position of the sensor. Hence, the origin is the same for all targets, but the coordinate frames have different angles $\varphi_{S n}$. This angle, as well as the distance $l_{S n}$, depend on the targets position in space. From Fig. 3 it is clear that,

$$
\begin{gathered}
x_{P_{4} O}^{R}+\left(l_{2}-l_{4}\right) \cos \varphi_{1}+l_{S n} \cos \varphi_{S n}-x_{P_{T n} O}^{R}=0, \\
y_{P_{4} O}^{R}+\left(l_{2}-l_{4}\right) \sin \varphi_{1}+l_{S n} \sin \varphi_{S n}-y_{P_{T n} O}^{R}=0 .
\end{gathered}
$$

Let us now define the relative angle to the leading vehicle,

$$
\delta_{S n} \triangleq \varphi_{S n}-\varphi_{1} \text {. }
$$


The road shape was described by (11) in the road frame $L_{3}$, where the $x$-axis is in the longitudinal direction of the vehicle. Differentiating (11) w.r.t. $x^{L_{3}}$ results in

$$
\frac{d y^{L_{3}}}{d x^{L_{3}}}=c_{0} x^{L_{3}}+\frac{c_{1}\left(x^{L_{3}}\right)^{2}}{2} .
$$

The Cartesian $x$-coordinate of the leading vehicle $P_{T n}$ in the $L_{3}$-frame is:

$$
x_{P_{T n} P_{3}}^{L_{3}}=x_{P_{T n} P_{1}}^{L_{1}}-l_{2}=l_{S n} \frac{\cos \delta_{S n}}{\cos \delta_{r}} .
$$

This gives us the angle of the leading vehicle relative to the road at $P_{3}$,

$$
\delta_{T n}=\varphi_{T n}-\varphi_{3}=\arctan \frac{d y^{L_{3}}}{d x^{L_{3}}} \text { for } x^{L_{3}}=x_{P_{T n} P_{3}}^{L_{3}}
$$

which is not absolutely correct, since the leading vehicle must not drive directly on the road line. However, it is sufficient for our purposes.

2) Kinematic Constraints: The target $T n$ is assumed to have zero lateral velocity, i.e., $\dot{y}^{L_{S n}}=0$. Furthermore, using the geometry of Fig. 1 we have

$$
-\dot{x}_{P_{T n} O}^{R} \sin \varphi_{S n}+\dot{y}_{P_{T n} O}^{R} \cos \varphi_{S n}=0 .
$$

3) Angle: The host vehicles velocity vector is applied in its $\mathrm{CoG} P_{4}$. The derivative of (20) is used together with (4) and (25) to get an expression for the derivative of the relative angle to the leading vehicle w.r.t. time

$$
\dot{\delta}_{S n}=-\frac{\dot{\varphi}_{1}\left(l_{2}-l_{4}\right) \cos \delta_{S n}+v_{x} \sin \left(\beta-\delta_{S n}\right)}{l_{S n}}-\dot{\varphi}_{1}
$$

\section{Resulting SENSOR FUSION Problem}

The resulting state-space model is divided into three parts, one for the host vehicle, one for the road and one for the leading vehicles, referred to as $H, R$ and $T$, respectively. In the final state-space model the three parts are augmented, resulting in a state vector of dimension $6+4 \cdot$ (Number of leading vehicles). Hence, the state vector varies with time, depending on the number of leading vehicles that we are currently tracking.

\section{A. Dynamic Motion Model}

We will in this section briefly summarize the dynamic motion models previously derived in Section II. The host vehicle model is described by the following states,

$$
x_{H}=\left(\begin{array}{lll}
\dot{\varphi}_{1} & \beta & l_{3}
\end{array}\right)^{T},
$$

i.e., the yaw rate, the float angle and the distance from the left lane marking. The corresponding differential equations were given in (5), (6) and (19), respectively.

The states describing the road $x_{R}$ are the road curvature at the host vehicle position $c_{0}$, the angle between the host vehicles direction of motion and the road curvature tangent $\delta_{R}$ and the width of the road $W$, i.e.,

$$
x_{R}=\left(\begin{array}{lll}
c_{0} & \delta_{R} & W
\end{array}\right)^{T} .
$$

The differential equations for $c_{0}$ and $\delta_{R}$ were given in (18) and (15), respectively. When it comes to the width of the current lane $W$, we simply make use of

$$
\dot{W}=0,
$$

motivated by the fact that $W$ does not change as fast as the other variables.

The states defining the targets are the azimuth angle $\delta_{S_{n}}$, the lateral position $l_{T n}$ of the target, the distance between the target and the host vehicle $l_{S n}$ and the relative velocity between the target and the host vehicle $i_{S n}$. This gives the following state vector for a leading vehicle

$$
x_{T}=\left(\begin{array}{llll}
\delta_{S n} & l_{T n} & i_{S n} & l_{S n}
\end{array}\right)^{T}
$$

The derivative of the azimuth angle was given in (26). It is assumed that the leading vehicles lateral velocity is small, implying that $\dot{l}_{T n}=0$ is a good assumption. Furthermore, it can be assumed that the leading vehicle accelerates similar to the host vehicle, thus $\ddot{l}_{S n}=0$ (compare with e.g., [6]).

Furthermore, the steering wheel angle $\delta_{F}$ and the host vehicle longitudinal velocity $v_{x}$ are modelled as input signals,

$$
u_{t}=\left(\begin{array}{ll}
\delta_{F} & v_{x}
\end{array}\right)^{T} .
$$

\section{B. Measurement Equations}

The measurement equation describes how the state variables relate to the measurements, i.e., it describes how the measurements enters the estimator. Recall that subscript $m$ is used to denote measurements. Let us start by introducing the measurements relating directly to the host vehicle motion, by defining

$$
y_{1}=\left(\begin{array}{ll}
\dot{\Psi} & a_{y, m}^{L_{4}}
\end{array}\right)^{T},
$$

where $\dot{\Psi}$ and $a_{y, m}^{C o G}$ are the measured yaw rate and the measured lateral acceleration, respectively. They are both measured with the host vehicles inertial sensor in the center of gravity. In order to find the corresponding measurement equation we start by observing that the host vehicle's lateral acceleration in the $\mathrm{CoG}$ is

$$
a_{y}^{L_{4}}=v_{x}(\dot{\varphi}+\dot{\beta})+\dot{v}_{x} \beta .
$$

Combining this expression with the centrifugal force and assuming $\dot{v}_{x} \beta=0$ yields

$$
\begin{aligned}
a_{y}^{L_{4}} & =v_{x}(\dot{\varphi}+\dot{\beta})=\beta \frac{-C_{\alpha f}-C_{\alpha r}-m \dot{v}_{x}}{m} \\
& +\dot{\varphi}_{1} \frac{-C_{\alpha f}\left(l_{1}-l_{4}\right)+C_{\alpha r} l_{4}}{m v_{x}}+\frac{C_{\alpha f}}{m} \delta_{F}
\end{aligned}
$$

Hence the measurement equations corresponding to (32) are given by

$$
h_{1}=\left(\beta \frac{-C_{\alpha f}-C_{\alpha r}-m \dot{v}_{x}}{m}+\dot{\varphi}_{1} \frac{\dot{\varphi}_{1}}{m C_{\alpha f}\left(l_{1}-l_{4}\right)+C_{\alpha r} l_{4}}-\frac{C_{\alpha f}}{m} \delta_{F}\right)
$$

The vision system provides measurements of the road geometry and the host vehicle position on the road according to

$$
y_{2}=\left(\begin{array}{llll}
c_{0, m} & \delta_{r, m} & W_{m} & l_{3, m}
\end{array}\right)^{T}
$$

and the corresponding measurement equations are given by

$$
\left.h_{2}=\left(\begin{array}{llll}
c_{0} & \left(\delta_{R}+\beta\right.
\end{array}\right) \quad W \quad l_{3}\right)^{T} .
$$

In order to include measurements of a leading vehicle we require that it is seen both by the radar and the vision system. The corresponding measurement vector is

$$
y_{3}=\left(\begin{array}{lll}
\delta_{S n, m} & i_{S n, m} & l_{S n, m}
\end{array}\right)^{T} .
$$


Since these are the state variable the measurement equation is obviously $h_{3}=\left(\begin{array}{lll}\delta_{S n} & i_{S n} & l_{S n}\end{array}\right)^{T}$. Finally, we have to introduce a nontrivial artificial measurement equation in order to reduce the drift in $l_{T n}$, and to introduce a further constraint on the road curvature. The measurement equation, which is derived from Fig. 3 is given by

$$
h_{4}=\frac{c_{0}\left(l_{S n} \cos \delta_{S n}\right)^{2}}{2}+\frac{l_{T n}}{\cos \delta_{T n}}+l_{3}+l_{S n}\left(\delta_{R}+\beta\right) \cos \delta_{S n},
$$

and the corresponding measurement is simply

$$
y_{4}=l_{S n, m} \sin \left(\delta_{S n, m}\right) .
$$

This might seem a bit ad hoc at first. However, the validity of the approach has recently been justified in the literature, see e.g., [20].

\section{Estimator}

The state-space model derived in the previous section is nonlinear and it is given in continuous time, whereas the measurements are in discrete time. The filtered estimates $\hat{x}_{t \mid t}$ are computed with an EKF. In order to do this we will first linearize and discretize the state-space model. This is a standard situation and a solid account of the underlying theory concerning this can be found in e.g., [11], [18].

The discretization is performed using the standard forward Euler method, resulting in $x_{t+T}=x_{t}+T f\left(x_{t}, u_{t}\right)=$ $g\left(x_{t}, u_{t}\right)$, where $T$ denotes the sample time. Now, at each time step the nonlinear state-space model is linearized by evaluating the Jacobian (i.e., the partial derivatives) of the $g\left(x_{t}, u_{t}\right)$-matrix at the current estimate $\hat{x}_{t \mid t}$. It is worth noting that this Jacobian is straightforwardly computed offline using symbolic software, such as MATHEMATICA.

The leading vehicles are estimated using rather standard techniques from target tracking, such as nearest neighbour data association and track counters in order to decide when to stop tracking a certain vehicle, etc. These are all important parts of the system we have implemented. However, since these techniques are rather standard we simply refer to the general treatments given in e.g., [1], [2].

\section{EXPERIMENTS AND RESUltS}

The experiments presented in this section are based on measurements acquired on public roads in Sweden during normal traffic circumstances. The host vehicle was equipped with radar and vision systems, measuring the distances and angles to the leading vehicles (targets). Information about the host vehicle motion, such as the steering wheel angle, yaw rate, etc. where acquired directly from the CAN bus. A more detailed description of the results and the method in general is provided in the accompanying technical report [15].

\section{A. Road Curvature Estimation}

The road curvature estimation using the sensor fusion approach shown in this paper is compared to a similar approach, thoroughly described in [6]. We refer to them as fusion 1 and fusion 2, respectively. Important differences between the two approaches are that in fusion 1 we model the float angle $\beta$ and make use of more information about the host vehicle motion. Furthermore, in fusion 2, the road is modelled according to (12) and a road aligned coordinate frame is used.

The curvature estimate $\hat{c}_{0}$ from the two sensor fusion approaches are compared to the estimate from the optical lane recognition (OLR) alone and a reference value (computed off-line using [7]). A typical result of this is shown in Fig. 5. The data stems from a country road, which explains the curvature values. It can be seen that the estimates

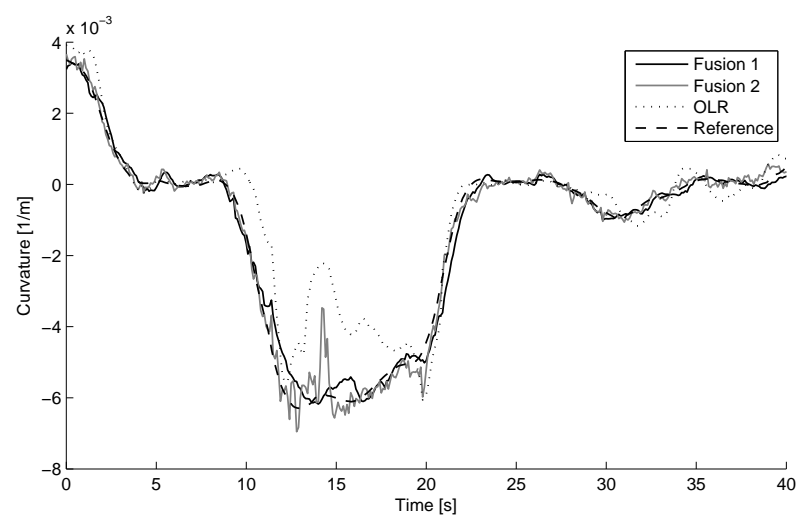

Fig. 5. Results from the two fusion approaches (solid black and gray lines) and the OLR (dotted line), showing the curvature estimate $\hat{c}_{0}$. As can be seen the curvature estimation can be improved by taking the other vehicles (gray line) and the host vehicle's driven curvature in account (solid black line). The dashed line is the reference curvature.

from the sensor fusion approaches gives better results than using the OLR alone, as was expected. The OLR estimate is rather noisy compared to the fused estimates. This is not surprising, since the pure OLR has less information. A second measurement sequence, acquired on a highway with two parallel lanes shows approximately the same result. To get a more aggregate view of the performance, we give the root mean square error (RMSE) for longer measurement sequences in Table I. Both fusion approaches improves the road curvature estimate by making use of the information about the leading vehicles, that is available from the radar and the vision systems. However, since we are interested in the curvature estimate also when there are no leading vehicles in front of the host vehicle this case will be studied as well. It is straightforward to study this case, it is just a matter of not providing the measurements of the leading vehicles to the algorithms. In Table I the RMSE values are provided for a few different scenarios. It is interesting to see that the advantage of fusion 1, which uses a more accurate host vehicle model, in comparison to fusion 2 is particularly noticeable when driving alone on a country road. The reason for this is first of all that there are no leading vehicles that could aid the fusion algorithm. Furthermore, the fact that we are driving on a rather curvy road implies that any additional information will help improving the curvature estimate. Here, the additional information is the improved host vehicle model used in fusion 1. The highway is rather straight and as

TABLE I

COMPARISON OF THE RMSE VALUES FOR THE TWO FUSION APPROACHES AND THE PURE MEASUREMENT (OLR) FOR TWO LONGER MEASUREMENT SEQUENCE ON PUBLIC ROADS. NOTE THAT ALL RMSE VALUES SHOULD BE MULTIPLIED BY $10^{-3}$.

\begin{tabular}{l|c|c|c|c}
\multicolumn{1}{c}{$\cdot 10^{-3}$} & \multicolumn{2}{c}{ Highway } & \multicolumn{2}{c}{ Country road } \\
\hline Time & \multicolumn{2}{c}{$15 \mathrm{~min}$} & \multicolumn{2}{c}{$9 \mathrm{~min}$} \\
OLR & \multicolumn{2}{c}{0.152} & \multicolumn{2}{c}{0.541} \\
\hline Leading vehicles & yes & no & yes & no \\
Fusion 1 (this paper) & 0.111 & 0.138 & 0.260 & 0.387 \\
Fusion 2 (method from [6]) & 0.126 & 0.143 & 0.266 & 0.499 \\
\hline
\end{tabular}


expected not much accuracy could be gained in using an improved dynamic vehicle model.

\section{B. Leading Vehicle Tracking}

A common problem with these road estimation methods is that it is hard to distinguish between the case when the leading vehicle is entering a curve and the case when the leading vehicle is performing a lane change. With the approach in this paper the information about the host vehicle motion, the OLR and the leading vehicles is weighted together in order to form an estimate of the road curvature. Fig. 6 shows an example from a situation on a three lane highway, where one

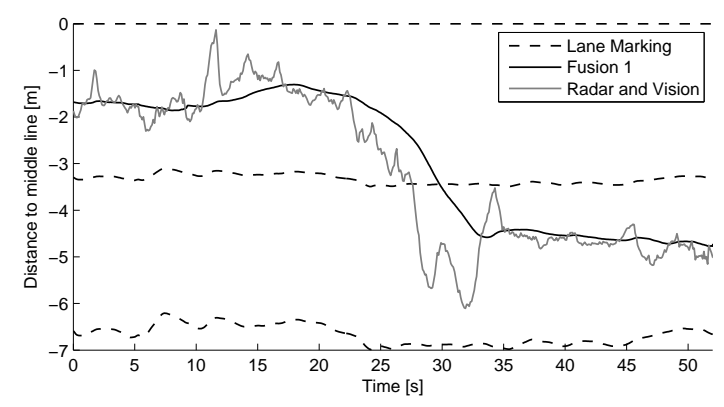

Fig. 6. Illustration of the lateral movement over time of the leading vehicle $l_{T n}$ driving on a highway with three lanes, where the leading vehicle changes lane. The estimate from our fusion approach is given by the solid black lines and the raw measurement signal is shown by the solid gray line. The dashed lines shows the lane markings. In this example the distance to the leading vehicle is $65 \mathrm{~m}$, see Fig. 7.

of the leading vehicles changes lane. The fusion approach in this paper produces an estimate of the lateral position of the leading vehicle which seems reasonably, but there is a time delay present in the estimate. To get a better understanding of this situation, one of the images acquired during the lane change is shown in Fig. 7.

For straight roads with several leading vehicles no difference between this and the second fusion approach mentioned above could be seen. This can be explained by the other leading vehicles, which stay in there lane and stabilizes the road geometry estimation.

\section{Conclusions}

We have presented a new formulation for the well studied problem of integrated road geometry estimation and vehicle tracking. The main differences to the existing approaches are that we have introduced a new dynamic model for the road and we make use of an improved host vehicle model.

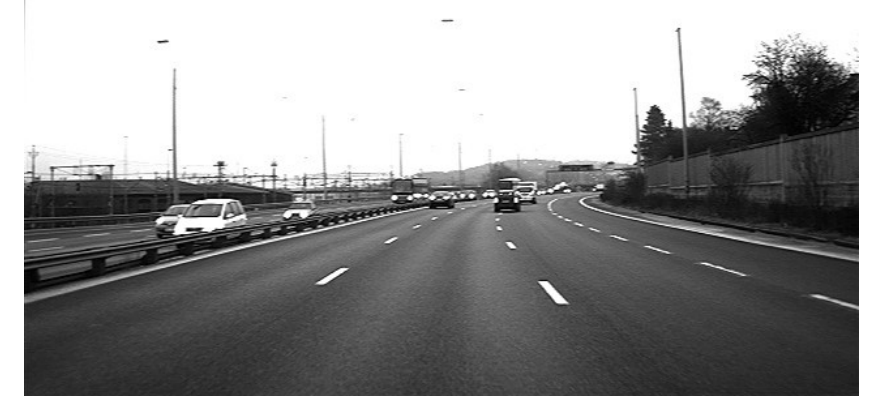

Fig. 7. Camera view for the situation in Fig. 6 during the lane change. The distance to the leading vehicle is approximately $65 \mathrm{~m}$.
The results obtained using measurements from real traffic situations clearly indicates that the gain in using the extended host vehicle model is most prominent when driving on country roads without any vehicles in front.

\section{ACKNOWLEDGEMENT}

The authors would like to thank Dr. Andreas Eidehall at Volvo Car Corporation for fruitful discussions. Furthermore, they would like to thank the SEnsor Fusion for Safety (SEFS) project within the Intelligent Vehicle Safety Systems (IVSS) program for financial support.

\section{REFERENCES}

[1] Y. Bar-Shalom, X. R. Li, and T. Kirubarajan, Estimation with Applications to Tracking and Navigation. New York: John Wiley \& Sons, 2001.

[2] S. S. Blackman and R. Popoli, Design and Analysis of Modern Tracking Systems. Norwood, MA, USA: Artech House, Inc., 1999.

[3] E. D. Dickmanns, Dynamic Vision for Perception and Control of Motion. Springer, 2007.

[4] E. D. Dickmanns and B. D. Mysliwetz, "Recursive 3-D road and relative ego-state recognition," IEEE Transactions on pattern analysis and machine intelligence, vol. 14, no. 2, pp. 199-213, Feb. 1992.

[5] E. D. Dickmanns and A. Zapp, "A curvature-based scheme for improving road vehicle guidance by computer vision," in Proceedings of the SPIE Conference on Mobile Robots, vol. 727, Cambridge, MA, USA, 1986, pp. 161-198.

[6] A. Eidehall, "Tracking and threat assessment for automotive collision avoidance," PhD thesis No 2007, Linköping Studies in Science and Technology, SE-581 83 Linköping, Sweden, Jan. 2007.

[7] A. Eidehall and F. Gustafsson, "Obtaining reference road geometry parameters from recorded sensor data," in Proceedings of the IEEE Intelligent Vehicles Symposium, Tokyo, Japan, Jun. 2006, pp. 256260.

[8] A. Eidehall, J. Pohl, and F. Gustafsson, "Joint road geometry estimation and vehicle tracking," Control Engineering Practice, vol. 15, no. 12 , pp. $1484-1494$, Dec. 2007

[9] A. Gern, U. Franke, and P. Levi, "Advanced lane recognition - fusing vision and radar," in Proceedings of the IEEE Intelligent Vehicles Symposimum, Dearborn, MI, USA, Oct. 2000, pp. 45-51.

[10] _ "Robust vehicle tracking fusing radar and vision," in Proceedings of the international conference of multisensor fusion and integration for intelligent systems, Baden-Baden, Germany, Aug. 2001, pp. 323-328.

[11] F. Gustafsson, Adaptive Filtering and Change Detection. New York, USA: John Wiley \& Sons, 2000.

[12] T. Kailath, A. H. Sayed, and B. Hassibi, Linear Estimation, ser. Information and System Sciences Series. Upper Saddle River, NJ, USA: Prentice Hall, 2000.

[13] U. Kiencke and L. Nielsen, Automotive Control Systems, 2nd ed. Berlin, Heidelberg, Germany: Springer, 2005.

[14] B. B. Litkouhi, A. Y. Lee, and D. B. Craig, "Estimator and controller design for lanetrak, a vision-based automatic vehicle steering system," in Proceedings of the 32nd IEEE Conference on Decision and Control, San Antonio, Texas, USA, Dec. 1993, pp. 1868 - 1873.

[15] C. Lundquist and T. B. Schön, "Road geometry estimation and vehicle tracking using a single track model," Department of Electrical Engineering, Linköping University, Sweden, Tech. Rep. LiTH-ISY-R2844, Mar. 2008.

[16] M. Mitschke and H. Wallentowitz, Dynamik der Kraftfahrzeuge, 4th ed. Berlin, Heidelberg: Springer, 2004.

[17] Robert Bosch, GmbH., Ed., Automotive Handbook, 6th ed. SAE Society of Automotive Engineers, 2004.

[18] W. J. Rugh, Linear System Theory, 2nd ed., ser. Information and system sciences series. Upper Saddle River, NJ, USA: Prentice Hall, 1996.

[19] T. B. Schön, A. Eidehall, and F. Gustafsson, "Lane departure detection for improved road geometry estimation," in Proceedings of the IEEE Intelligent Vehicle Symposium (IV), Tokyo, Japan, Jun. 2006, pp. 546551.

[20] B. O. S. Teixeira, J. Chandrasekar, L. A. B. Torres, L. A. Aguirre, and D. S. Bernstein, "State estimation for equality-constrained linear systems," in Proceedings of the 46th Conference on Decision and Control (CDC), New Orleans, LA, USA, Dec. 2007, pp. 6220-6225.

[21] Z. Zomotor and U. Franke, "Sensor fusion for improved vision based lane recognition and object tracking with range-finders," in Proceedings of IEEE Conference on Intelligent Transportation System, Boston, MA, USA, Nov. 1997, pp. 595-600. 